\title{
ON MULTIDIMENSIONAL SINC-GAUSS SAMPLING FORMULAS FOR ANALYTIC FUNCTIONS*
}

\author{
RASHAD M. ASHARABI ${ }^{\dagger}$ AND FELWAH H. AL-HADDAD ${ }^{\dagger}$
}

\begin{abstract}
Using complex analysis, we present new error estimates for multidimensional sinc-Gauss sampling formulas for multivariate analytic functions and their partial derivatives, which are valid for wide classes of functions. The first class consists of all $n$-variate entire functions of exponential type satisfying a decay condition, while the second is the class of $n$-variate analytic functions defined on a multidimensional horizontal strip. We show that the approximation error decays exponentially with respect to the localization parameter $N$. This work extends former results of the first author and J. Prestin, [IMA J. Numer. Anal., 36 (2016), pp. 851-871] and [Numer. Algorithms, 86 (2021), pp. 1421-1441], on two-dimensional sinc-Gauss sampling formulas to the general multidimensional case. Some numerical experiments are presented to confirm the theoretical analysis.
\end{abstract}

Key words. multidimensional sinc-Gauss sampling formula, multivariate analytic function, localization operator, error estimate

AMS subject classifications. 94A20, 32A15, 41A25, 41A80

1. Introduction. During the past two decades, the modification of various types of univariate and bivariate sampling series using Gaussian multipliers has attracted many researchers due to the slow convergence rate of these types of sampling. The modification of univariate sampling with Gaussian multipliers was studied using a Fourier-analytic approach by Qian and his collaborators; see, e.g., [12, 13, 14, 15]. Schmeisser and Stenger in [18], Tanaka et al. in [19], Asharabi in [2], and Asharabi and Prestin in [6] have studied the modification of various types of univariate sampling series based on a complex-analytic approach. The modification of bivariate classical and Hermite sampling with bivariate Gaussian multipliers is introduced in [3, 6] based on a complex-analytic approach. Furthermore, these modifications are employed for approximating the ordinary and partial derivatives of analytic functions with high accuracy; cf. [4, 7]. To the best of our knowledge, there is no single study on the modification of the general multivariate sampling series with Gaussian multipliers, the work of Lin and Zhang in [10] being an exception. They have studied their modification based on a Fourier-analytic approach.

Now let us briefly review the general multivariate Whittaker-Kotelnikov-Shannon (WKS) sampling series and the modification of Lin and Zhang. The Bernstein space $B_{\sigma}^{p}\left(\mathbb{R}^{n}\right), \sigma>0$, $1 \leq p<\infty$, is the set of all functions from $L^{p}\left(\mathbb{R}^{n}\right)$ that can be extended to $n$-variate entire functions of exponential type $\sigma$, where $L^{p}\left(\mathbb{R}^{n}\right)$ denotes the Banach space of all complexvalued Lebesgue-measurable $n$-variate functions with the standard norm

$$
\|f\|_{p}= \begin{cases}\left(\int_{\mathbb{R}^{n}}|f(x)|^{p} d x\right)^{1 / p} & 1 \leq p<\infty \\ \text { ess. } \sup _{x \in \mathbb{R}^{n}}|f(x)| & p=\infty\end{cases}
$$

According to Schwartz's theorem [11, p. 109], the Bernstein space is defined by

$$
B_{\sigma}^{p}\left(\mathbb{R}^{n}\right)=\left\{f \in L^{p}\left(\mathbb{R}^{n}\right): \quad \operatorname{supp} \widehat{f} \subset[-\sigma, \sigma]^{n}\right\}
$$

* Received June 9, 2021. Accepted November 11, 2021. Published online on January 10, 2022. Recommended by Daniel Potts.

${ }^{\dagger}$ Department of Mathematics, College of Arts and Sciences, Najran University, Najran, Saudi Arabia, ( $\{$ rashad1974, felwah-4994\}@hotmail.com). 


\section{ETNA}

Kent State University and

Johann Radon Institute (RICAM)

where $\widehat{f}$ is the Fourier transform of $f$ in the sense of generalized functions. The general multivariate WKS sampling theorem states that if $f$ belongs to the Bernstein space $B_{\sigma}^{p}\left(\mathbb{R}^{n}\right)$, $1 \leq p<\infty$, then $f$ can be represented as

$$
f(z)=\sum_{k \in \mathbb{Z}^{n}} f\left(\frac{k \pi}{\sigma}\right) \prod_{j=1}^{n} \operatorname{sinc}\left(\sigma z_{j}-k_{j} \pi\right),
$$

where $k=\left(k_{1}, \ldots, k_{n}\right) \in \mathbb{Z}^{n}, z:=\left(z_{1}, \ldots, z_{n}\right) \in \mathbb{C}^{n}$, and the sinc function is defined as

$$
\operatorname{sinc}(t):= \begin{cases}\frac{\sin t}{t} & t \neq 0 \\ 1 & t=0\end{cases}
$$

The series (1.2) converges absolutely and uniformly on $\mathbb{R}^{n}$ and uniformly on any compact subset of $\mathbb{C}^{n}$; see, e.g., [22]. The convergence rate for this series is slow unless $|f(x)|$ decays rapidly as $\left|x_{j}\right| \rightarrow \infty$ for all $1 \leq j \leq n$, and it is of order $O\left(N^{-1 / p}\right), p>1$; cf. [23]. Here, $N$ denotes the number of samples of $f$ in the truncated series. The slow convergence rate is due to the slow decay of the sinc function. Asharabi and Al-Abbas in [5] have incorporated a convergence factor from the Bernstein space $B_{a}^{p}\left(\mathbb{R}^{n}\right)$ into the sampling series (1.2), and they have established a regularized multivariate sampling expansion. Let $f \in B_{(1-\theta) \sigma}^{p}\left(\mathbb{R}^{n}\right)$, $0<\theta<1,1 \leq p<\infty$, and $\prod_{j=1}^{n} \psi_{j} \in B_{\theta \sigma}^{p}\left(\mathbb{R}^{n}\right)$. Then, for $x \in \mathbb{R}^{n}$, we have [5]

$$
f(x)=\sum_{k \in \mathbb{Z}^{n}} f(k h) \prod_{j=1}^{n} \psi_{j}\left(h^{-1} x_{j}-k_{j}\right) \operatorname{sinc}\left(\pi h^{-1} x_{j}-k_{j} \pi\right),
$$

where $\psi_{j}(0)=1, j=1, \ldots, n$, and $h \in(0, \pi / \sigma]$. The series (1.4) converges absolutely and uniformly on $\mathbb{R}^{n}$ and on any compact subset of $\mathbb{C}^{n}$. Its convergence rate depends on the convergence factor $\left\{\psi_{j}\right\}_{j=1}^{n}$. According to Nikol'skii, the decay of $B_{\sigma}^{p}\left(\mathbb{R}^{n}\right)$-functions is limited. It is well-known that the function in the space $B_{\sigma}^{p}\left(\mathbb{R}^{n}\right)$ cannot decay faster in $\mathbb{R}^{n}$ than $\prod_{i=1}^{n} e^{-a_{i}\left|x_{i}\right|^{m_{i}}}$ for $\left|x_{i}\right| \rightarrow \infty$, where $\left.x:=\left(x_{1}, \ldots, x_{n}\right), m_{j} \in\right] 0,1\left[, a_{i}\right.$ are positive numbers, and $j=1, \ldots, n$. Here we would like to mention that the modification of the multivariate WKS sampling series (1.2) with a bandlimited multiplier can reconstruct the functions exactly; see (1.4). If we modify the sampling series with a non-bandlimited multiplier, like a Gaussian multiplier, then the modification formula cannot reconstruct the function exactly.

Lin and Zhang have defined their modification for the multivariate WKS sampling (1.2) as, cf. [10],

$$
S_{\sigma, N}[f](x)=\sum_{k \in J_{N}} f(k) \prod_{j=1}^{n} \operatorname{sinc}\left(\pi x_{j}-k_{j} \pi\right) \exp \left(\frac{-\delta\left(x_{j}-j\right)^{2}}{N-2}\right),
$$

where $N \geq 2, \delta=(\pi-\sigma) / 2, \sigma \in(0, \pi)$, and $J_{N}:=\left\{k \in \mathbb{Z}^{n}: k \in(-N, N]^{n}\right\}$. The formula (1.5) is used to reconstruct a multivariate function $f$ that belongs to the Paley-Wiener space $B_{\sigma}^{2}\left(\mathbb{R}^{n}\right)$ from its finite samples $f(k), k \in J_{N}$. Based on a Fourier-analytic approach, they have estimated the error $\left|f(x)-S_{\sigma, N}[f](x)\right|$ for $f \in B_{\sigma}^{2}\left(\mathbb{R}^{n}\right)$ by a bound of exponential order. In other word, if $f \in B_{\sigma}^{2}\left(\mathbb{R}^{n}\right)$, then for $x \in(0,1)^{n}$, Lin and Zhang have shown in [10, Theorem 2.5] that

$$
\left|f(x)-S_{\sigma, N}[f](x)\right|=O\left(\frac{e^{-\delta(N-1)}}{\sqrt{N-1}}\right) .
$$


The bound in (1.6) is valid only for real-valued functions from the Paley-Wiener space $B_{\sigma}^{2}\left(\mathbb{R}^{n}\right)$ and needs an adaptation when we approximate the function $f$ outside the region $(0,1)^{n}$. Here we would like to point out that the modification of the sampling series with Gaussian multipliers, which uses samples only from the function itself, is called sinc-Gauss formula; cf., e.g., [4, 19, 21].

In this paper, based on the complex-analytic approach, we modify the general multivariate WKS sampling (1.2) with the help of a Gaussian multiplier. This modification will be valid for wide classes of functions. The first class consists of all $n$-variate entire functions of exponential type satisfying a decay condition, while the second is the class of $n$-variate analytic functions defined on a multidimensional horizontal strip. Also, this modification extends the results in $[6,7]$ to multidimensional sinc-Gaussian sampling formulas. Asharabi and Prestin, cf. [6, 7], have established only the bivariate sinc-Gauss sampling formula and left the extension of their results to the general multidimensional case as an open problem. They have shown that a straightforward generalization to the general multivariate case for their kernel does not lead to the desired estimate, cf. [7, Remark 2.2.]. Here we have found a meaningful way to transfer the bivariate kernel to the general multivariate case. All the results in $[6,7]$ will be extended to the general multivariate case.

The rest of the paper is organized as follows: In Section 2, we establish the multivariate sinc-Gauss sampling formula and estimate the error for entire and holomorphic functions on an infinite multivariate horizontal strip domain. Section 3 is devoted to applying the multivariate sinc-Gauss formula to approximate the partial derivatives of analytic function of several variables of any order using only finitely many samples of the function itself. Section 4 deals with numerical illustrations.

2. Multivariate sinc-Gauss sampling. Let $E_{\sigma}^{n}(\varphi), \sigma>0$, be the class of entire functions of several variables satisfying the following growth condition

$$
|f(z)| \leq \varphi\left(\left|\Re z_{1}\right|, \ldots,\left|\Re z_{n}\right|\right) \exp \left(\sigma \sum_{j=1}^{n}\left|\Im z_{j}\right|\right), \quad z:=\left(z_{1}, \ldots, z_{n}\right) \in \mathbb{C}^{n}
$$

where $\varphi$ is a non-negative function on $\mathbb{R}_{+}^{n}$ that is nondecreasing for all variables $\left|\Re z_{j}\right|$, $j=1, \ldots, n$. The authors of [6] have introduced the space $E_{\sigma}^{2}(\varphi)$ and showed the inclusion $B_{\sigma}^{p}\left(\mathbb{R}^{n}\right) \subset E_{\sigma}^{n}(\varphi), n=2$. This inclusion is still true for all $n>2$. For $h \in(0, \pi / \sigma]$, set $\beta:=(\pi-h \sigma) / 2$, and let $E^{n}$ be the class of all entire functions on $\mathbb{C}^{n}$. In the class $E_{\sigma}^{n}(\varphi)$, we define the multivariate localization operator $\mathcal{G}_{n, h, N}: E_{\sigma}^{n}(\varphi) \rightarrow E^{n} \cap L^{p}\left(\mathbb{R}^{n}\right)$ as follows:

$$
\mathcal{G}_{n, h, N}[f](z):=\sum_{k \in \mathbb{Z}_{N}^{n}(z)} f(k h) \prod_{j=1}^{n} \operatorname{sinc}\left(\pi h^{-1} z_{j}-k_{j} \pi\right) \exp \left(-\frac{\beta\left(z_{j}-k_{j} h\right)^{2}}{N h^{2}}\right),
$$

where $N$ is a positive integer, $z \in \mathbb{C}^{n}, k=\left(k_{1}, \ldots, k_{n}\right)$, and

$$
\mathbb{Z}_{N}^{n}(z):=\left\{k \in \mathbb{Z}^{n}:\left|\left\lfloor h^{-1} \Re z_{j}+1 / 2\right\rfloor-k_{j}\right| \leq N, j=1, \ldots, n\right\} .
$$

The univariate and bivariate case of the operator $\mathcal{G}_{n, h, N}$ are introduced in [18] and [6], respectively. For establishing the operator $\mathcal{G}_{n, h, N}$, we need to consider the kernel function

$$
\mathcal{K}_{z}(w):=\rho_{z}(w) \prod_{j=1}^{n} \frac{\exp \left(-\beta\left(z_{j}-w_{j}\right)^{2} / N h^{2}\right)}{\left(w_{j}-z_{j}\right) \sin \left(\pi h^{-1} w_{j}\right)}
$$


where $w:=\left(w_{1}, \ldots, w_{n}\right), z=\left(z_{1}, \ldots, z_{n}\right)$, and

$$
\rho_{z}(w):=-\prod_{j=1}^{n}\left(\sin \left(\pi h^{-1} w_{j}\right)-\sin \left(\pi h^{-1} z_{j}\right)\right)+\prod_{j=1}^{n} \sin \left(\pi h^{-1} w_{j}\right) .
$$

The bivariate form of this kernel function is considered in [6]. As we have mentioned in the introduction, the authors of [7] have shown that a straightforward generalization for their bivariate kernel does not lead to the multivariate operator $\mathcal{G}_{n, h, N}$; cf. [7, Remark 2.2.]. In (2.4) and (2.5), we found a meaningful way to transfer their bivariate kernel to the general multivariate case. On the $w_{j}$-plane, let $R_{j}$ be the positively oriented rectangle with vertices at $\pm h\left(N+\frac{1}{2}\right)+h\left\lfloor h^{-1} \Re z_{j}+\frac{1}{2}\right\rfloor+\mathrm{i}\left(\Im z_{j} \pm N\right)$, for all $j=1, \ldots, n$. This kernel has a singularity of order 1 at all the points of the sets

$$
\left\{w \in \mathbb{C}^{n}: w_{j}=z_{j} \text { for some } j\right\} \cup\left\{w \in \mathbb{C}^{n}: w_{j}=k_{j} h \text { for some } j\right\} .
$$

In the following result, we show that the error of the approximation by the operator $\mathcal{G}_{n, h, N}$ for functions from $E_{\sigma}^{n}(\varphi)$ can be written as the integral of the kernel $\mathcal{K}_{z}$ over the rectangles $R_{1}, \ldots, R_{n}$.

LEMMA 2.1. Let $f \in E_{\sigma}^{n}(\varphi)$. Then we have for all $z \in \mathbb{C}^{n}$

$$
f(z)-\mathcal{G}_{n, h, N}[f](z)=\frac{1}{(2 \pi \mathrm{i})^{n}} \oint_{R_{n}} \ldots \oint_{R_{1}} \mathcal{K}_{z}(w) f(w) d w_{1} \ldots d w_{n}
$$

where $R_{j}, j=1, \ldots, n$, is the positively oriented rectangle defined above.

Proof. For convenience, we define $F(w):=\mathcal{K}_{z}(w) f(w)$. Let $\operatorname{Res}_{1} F\left(\lambda_{1} ; w_{2}, \ldots, w_{n}\right)$ be the residue of the univariate function $w_{1} \mapsto F\left(w_{1}, \ldots, w_{n}\right)$ at the point $w_{1}=\lambda_{1} \in \mathbb{C}$ with $w_{2}, \ldots, w_{n}$ considered complex parameters. Assume that

$$
\operatorname{Res}_{\ell-1} F\left(\lambda_{1}, \ldots, \lambda_{\ell-1} ; w_{\ell}, \ldots, w_{n}\right)
$$

has already been defined. Then $\operatorname{Res}_{\ell} F\left(\lambda_{1}, \ldots, \lambda_{\ell} ; w_{\ell+1}, \ldots, w_{n}\right)$ is defined as the residue of the univariate function $w_{\ell} \mapsto \operatorname{Res}_{\ell-1} F\left(\lambda_{1}, \ldots, \lambda_{\ell-1} ; w_{\ell}, \ldots, w_{n}\right)$ at the point $w_{\ell}=\lambda_{\ell} \in \mathbb{C}$ with $w_{\ell+1}, \ldots, w_{n}$ considered complex parameters. By the special form of the function $F$, it can be verified that

$$
\operatorname{Res}_{\ell} F\left(\lambda_{1}, \ldots, \lambda_{\ell} ; w_{\ell+1}, \ldots, w_{n}\right) \equiv 0 \quad \text { unless } \quad\left(\lambda_{1}, \ldots, \lambda_{\ell}\right) \in\left\{\left(z_{1}, \ldots, z_{\ell}\right)\right\} \cup h \mathbb{Z}^{\ell},
$$

where $2 \leq \ell \leq n$. Furthermore, we have for all $z \in \mathbb{C}^{n}$

$$
\operatorname{Res}_{n} F\left(z_{1}, \ldots, z_{n}\right)=f(z)
$$

and

$$
\operatorname{Res}_{n} F\left(k_{1} h, \ldots, k_{n} h\right)=-f(k h) \prod_{j=1}^{n} \operatorname{sinc}\left(\pi h^{-1} z_{j}-k_{j} \pi\right) \exp \left(-\frac{\beta\left(z_{j}-k_{j} h\right)^{2}}{N h^{2}}\right)
$$

for all $\left(k_{1}, \ldots, k_{n}\right) \in \mathbb{Z}^{n}$. Calculating the integrals on the right-hand side of (2.6) one after the other with the help of the residue theorem, we get

$$
\begin{aligned}
\frac{1}{(2 \pi \mathrm{i})^{n}} \oint_{R_{n}} \ldots \oint_{R_{1}} \mathcal{K}_{z}(w) f(w) d w_{1} \ldots d w_{n}=\operatorname{Res}_{n} F\left(z_{1}, \ldots, z_{n}\right) \\
+\sum_{k \in \mathbb{Z}_{N}^{n}(z)} \operatorname{Res}_{n} F\left(k_{1} h, \ldots, k_{n} h\right) .
\end{aligned}
$$


Combining (2.7), (2.8), and (2.9) implies (2.6).

In the following result, we rewrite the function $\rho_{z}(w)$, which is defined by (2.5), as a finite sum of products. This formula will be used in the proof of Theorem 2.3.

LEMMA 2.2. The function $\rho_{z}(w)$ can be written in the form

$$
\rho_{z}(w)=\sum_{m=1}^{n}(-1)^{m+1} \sum_{N_{m} \in P(I)} \prod_{j \in N_{m}} \sin \left(\pi h^{-1} z_{j}\right) \prod_{l \in M_{m}} \sin \left(\pi h^{-1} w_{l}\right),
$$

where $N_{m}$ is a subset of $I=\{1,2, \ldots, n\}$ such that it contains any melements, $M_{m}=I \backslash N_{m}$, and $P(I)$ is the set of all subsets of $I$. Note that $M_{n}$ is the empty set.

Proof. The proof can be easily established by mathematical induction, so we leave it to the reader.

In the following theorem, we estimate the integral in (2.6) to find an error bound for $\left|f(z)-\mathcal{G}_{n, h, N}[f](z)\right|$.

THEOREM 2.3. Let $f \in E_{\sigma}^{n}(\varphi)$. Then we have for all $\left|\Im z_{j}\right|<N, j=1, \ldots, n$,

$$
\left|f(z)-\mathcal{G}_{n, h, N}[f](z)\right| \leq 2 \varphi(\eta(z)) B_{N}(z) \frac{e^{-\beta N}}{\sqrt{\pi \beta N}}
$$

where the function $\varphi$ is given in (2.1) and $\eta$ is defined as

$$
\eta(z):=\left(b\left(z_{1}\right), \ldots, b\left(z_{n}\right)\right)
$$

such that $b\left(z_{j}\right):=\left|\Re z_{j}\right|+h(N+1)$, for all $j=1, \ldots, n$. The function $B_{N}$ is defined by

$$
B_{N}(z):=\sum_{m=1}^{n} \prod_{l \in M_{m}} e^{\sigma\left|\Im z_{l}\right|}\left(\frac{2 e^{-\beta N}}{\sqrt{\pi \beta N}}\right)^{m-1} \sum_{N_{m} \in P(I)} \prod_{j \in N_{m}}\left|\sin \left(\pi h^{-1} z_{j}\right)\right| \theta_{N}\left(h^{-1} \Im z_{j}\right),
$$

where

$$
\begin{aligned}
\theta_{N}(t) & :=\cosh (2 \beta t)+\frac{2 e^{\beta t^{2} / N}}{\sqrt{\pi \beta N}\left(1-(t / N)^{2}\right)}+\frac{1}{2}\left[\frac{e^{2 \beta t}}{e^{2 \pi(N-t)}-1}+\frac{e^{-2 \beta t}}{e^{2 \pi(N+t)}-1}\right] \\
\text { (2.14) } & =\cosh (2 \beta t)+O\left(N^{-1 / 2}\right) \quad \text { as } N \rightarrow \infty .
\end{aligned}
$$

Proof. Substituting (2.4) and (2.10) into (2.6), the integral in (2.6) can be written in the form

$$
\begin{aligned}
& f(z)-\mathcal{G}_{n, h, N}[f](z) \\
&=\frac{1}{(2 \pi \mathrm{i})^{n}}\left[\sum_{m=1}^{n}(-1)^{m+1} \sum_{N_{m} \in P(I)} \prod_{j \in N_{m}} \sin \left(\pi h^{-1} z_{j}\right)\right. \\
&\left.\quad \times \oint_{R_{n}} \ldots \oint_{R_{1}} \frac{f(w) \prod_{j \in N_{m}} e^{-\beta\left(z_{j}-w_{j}\right)^{2} / N h^{2}} \prod_{l \in M_{m}} e^{-\beta\left(z_{l}-w_{l}\right)^{2} / N h^{2}}}{\prod_{j \in N_{m}} \sin \left(\pi h^{-1} w_{j}\right)\left(w_{j}-z_{j}\right) \prod_{l \in M_{m}}\left(w_{l}-z_{l}\right)} d w_{1} \ldots d w_{n}\right],
\end{aligned}
$$

where $R_{j}, j=1, \ldots, n$, is the positively oriented rectangle defined above. Applying the multidimensional version of the Cauchy integral formula, cf., e.g., [9, p. 26], we get for a 
fixed $m$,

$$
\begin{aligned}
& \left\{|f(w)|_{w_{l}=z_{l}}\right\}_{l \in M_{m}} \\
& \quad=\frac{1}{(2 \pi \mathrm{i})^{n-m}} \oint_{\prod_{l \in M_{m}} R_{l}} \frac{f(w) \prod_{l \in M_{m}} \exp \left(-\beta\left(z_{l}-w_{l}\right)^{2} / N h^{2}\right)}{\prod_{l \in M_{m}}\left(w_{l}-z_{l}\right)} \prod_{l \in M_{m}} d w_{l},
\end{aligned}
$$

where $z_{\ell} \in \mathbb{C}, \ell \in M_{m}$, are arbitrary but fixed complex parameters, whereas $w_{\ell} \in \mathbb{C}, \ell \in M_{m}$, are complex variables. Combining (2.16), (2.15), and in view of the definition of the sets $M_{m}$ and $N_{m}$, we obtain

$$
\begin{aligned}
& \left|f(z)-\mathcal{G}_{n, h, N}[f](z)\right| \\
& \leq \sum_{m=1}^{n}\left[\frac{1}{(2 \pi)^{m}} \sum_{N_{m} \in P(I)} \prod_{j \in N_{m}}\left|\sin \left(\pi h^{-1} z_{j}\right)\right|\right. \\
& \left.\times \oint_{\prod_{j \in N_{m}} R_{j}}\left|\frac{\left\{\left.f(w)\right|_{w_{l}=z_{l}}\right\}_{l \in M_{m}} \prod_{j \in N_{m}} \exp \left(-\beta\left(z_{j}-w_{j}\right)^{2} / N h^{2}\right)}{\prod_{j \in N_{m}} \sin \left(\pi h^{-1} w_{j}\right)\left(w_{j}-z_{j}\right)}\right| \prod_{j \in N_{m}}\left|d w_{j}\right|\right] .
\end{aligned}
$$

Since $f \in E_{\sigma}^{n}(\varphi)$ for all points $w \in \prod_{j=1}^{n} R_{j}$, we have

$$
|f(w)| \leq \varphi(\eta(z)) \prod_{j=1}^{n} e^{\sigma\left|\Im w_{j}\right|},
$$

where the function $\eta$ is defined in (2.12). Aside from that, it is easy to verify that for all $w_{j} \in R_{j}$, we have

$$
\left|\left\{\left.f(w)\right|_{w_{l}=z_{l}}\right\}_{l \in M_{m}}\right| \leq \varphi(\eta(z)) \prod_{j \in N_{m}} e^{\sigma\left|\Im w_{j}\right|} \prod_{l \in M_{m}} e^{\sigma\left|\Im z_{l}\right|},
$$

for all $M_{m} \subset I$. This results from the assumption that the function $\varphi$ is nondecreasing for all variables $\left|\Re z_{j}\right|, j=1, \ldots, n$. Substituting (2.18) and (2.19) into (2.17), we obtain

$$
\begin{aligned}
\left|f(z)-\mathcal{G}_{n, h, N}[f](z)\right| & \\
\leq \varphi(\eta(z)) \sum_{m=1}^{n} & {\left[\frac{1}{(2 \pi)^{m}} \sum_{N_{m} \in P(I)} \prod_{j \in N_{m}}\left|\sin \left(\pi h^{-1} z_{j}\right)\right| \prod_{l \in M_{m}} e^{\sigma\left|\Im z_{l}\right|}\right.} \\
& \left.\times \prod_{j \in N_{m}} \oint_{R_{j}}\left|\frac{\exp \left(\sigma\left|\Im w_{j}\right|-\beta\left(z_{j}-w_{j}\right)^{2} / N h^{2}\right)}{\sin \left(\pi h^{-1} w_{j}\right)\left(w_{j}-z_{j}\right)}\right|\left|d w_{j}\right|\right] .
\end{aligned}
$$

By splitting the contour integral over $R_{j}$ into four integrals along line segments and transforming the latter into ordinary integrals, one can imitate the estimates in [18, pp. 203-205] to obtain

$$
\oint_{R_{j}}\left|\frac{\exp \left(\sigma\left|\Im w_{j}\right|-\beta\left(z_{j}-w_{j}\right)^{2} / N h^{2}\right)}{\sin \left(\pi h^{-1} w_{j}\right)\left(w_{j}-z_{j}\right)}\right|\left|d w_{j}\right| \leq 4 \pi \theta_{N}\left(h^{-1} \Im z_{j}\right) \frac{e^{-\beta N}}{\sqrt{\pi \beta N}} .
$$

Combining (2.21) and (2.20), we finally get (2.11). 
REMARK 2.4. The special cases of Theorem 2.3 for $n=1$ and $n=2$ were stated by Schmeisser and Stenger in [18, Theorem 2.1] and by Asharabi and Prestin in [6, Theorem 3.3], respectively.

On the complex domain, the bound in (2.11) depends on the growth of the functions $\varphi$ and $B_{N}$, while on the real domain, the bound (2.11) depends only on the growth of $\varphi$. This holds because the function $B_{N}$ is a bounded function on the real domain. Precisely, for all $x \in \mathbb{R}^{n}$, we have

$$
\left|B_{N}(x)\right| \leq \sum_{m=1}^{n}\left(\begin{array}{c}
n \\
m
\end{array}\right)\left(\frac{2 e^{-\beta N}}{\sqrt{\pi \beta N}}\right)^{m-1} \theta_{N}^{m}(0) .
$$

Since the function $\varphi$ is nondecreasing, we can take one of familiar growth: constant, polynomial, and exponential growth. In the following results, we investigate the cases of slower and faster growth, which will be advantageous special cases of Theorem 2.3.

COROLlaRY 2.5. Assume that $f \in B_{\sigma}^{\infty}\left(\mathbb{R}^{n}\right)$. Then for all $z \in \mathbb{C}^{n}$ we have

$$
\left|f(z)-\mathcal{G}_{n, h, N}[f](z)\right| \leq 2\|f\|_{\infty} B_{N}(z) \frac{e^{-\beta N}}{\sqrt{\pi \beta N}} .
$$

If $f$ belongs to the Paley-Wiener space $B_{\sigma}^{2}\left(\mathbb{R}^{n}\right)$, then we get for all $z \in \mathbb{C}^{n}$ that

$$
\left|f(z)-\mathcal{G}_{n, h, N}[f](z)\right| \leq 2(\sigma / \pi)^{\frac{n}{2}}\|f\|_{2} B_{N}(z) \frac{e^{-\beta N}}{\sqrt{\pi \beta N}},
$$

where the norm $\|f\|_{p}, 1 \leq p \leq \infty$, is defined by (1.1) and $B_{N}$ is given in (2.13).

Proof. Since $f \in B_{\sigma}^{\infty}\left(\mathbb{R}^{n}\right)$, it satisfies the growth condition

$$
|f(z)| \leq\|f\|_{\infty} \prod_{j=1}^{n} e^{\sigma\left|\Im z_{j}\right|},
$$

and the decay condition in (2.1) is valid with the constant growth $\varphi:=\|f\|_{\infty}$. Note that the norm $\|f\|_{\infty}$ is defined by (1.1). Therefore, we get (2.22). For $f \in B_{\sigma}^{2}\left(\mathbb{R}^{n}\right)$, we have

$$
f(x)=\frac{1}{(2 \pi)^{n / 2}} \int_{[-\sigma, \sigma]^{n}} \hat{f}(w) e^{\mathrm{i}\langle x, w\rangle} d w
$$

where $\hat{f}$ is the Fourier transform of $f$ and $\langle x, w\rangle$ is the inner product on $\mathbb{R}^{n}$. Applying $n$-times the CauchyâĂŞSchwarz inequality yields

$$
\|f\|_{\infty} \leq \frac{1}{(2 \pi)^{n / 2}} \int_{[-\sigma, \sigma]^{n}}|\hat{f}(w)| d w \leq(\sigma / \pi)^{\frac{n}{2}}\|\hat{f}\|_{2}
$$

Since $f \in L^{2}\left(\mathbb{R}^{n}\right)$, the Parseval equality holds, cf., e.g., [20, p. 292],

$$
\|\hat{f}\|_{2}=\|f\|_{2}
$$

The proof is completed by combining (2.26), (2.25), and (2.22) and using the fact that $B_{\sigma}^{2}\left(\mathbb{R}^{n}\right) \subset B_{\sigma}^{\infty}\left(\mathbb{R}^{n}\right)$.

COROLlary 2.6. Assume that $f$ is an entire function satisfying the exponential growth condition

$$
|f(z)| \leq M \prod_{j=1}^{n} e^{\kappa\left|\Re z_{j}\right|+\sigma\left|\Im z_{j}\right|}, \quad z \in \mathbb{C}^{n}, M>0
$$


where $\sigma, \kappa$ are non-negative numbers and $\sigma+\kappa>0$. Then, we have for $\left|\Im z_{j}\right|<N$ that

$$
\left|f(z)-\mathcal{G}_{n, h, N}[f](z)\right| \leq 2 M e^{\kappa\left(h+\sum_{j=1}^{n}\left|\Re z_{j}\right|\right)} B_{N}(z) \frac{e^{-(\beta-\kappa h) N}}{\sqrt{\pi \beta N}},
$$

where $h \in(0, \pi /(\sigma+2 \kappa))$ and $B_{N}$ is defined in (2.13).

Proof. By assumption, $f$ is an entire function satisfying (2.27). In this case, we get

$$
\left|\left\{\left.f(w)\right|_{w_{l}=z_{l}}\right\}_{l \in M_{m}}\right| \leq M\left[e^{\kappa h(N+1)}\right]^{m} e^{\kappa\left(\sum_{j=1}^{n}\left|\Re z_{j}\right|\right)} \prod_{l \in M_{m}} e^{\sigma\left|\Im z_{l}\right|} \prod_{j \in N_{m}} e^{\sigma\left|\Im w_{j}\right|} .
$$

Using the estimate in the proof of Theorem 2.3, we get (2.28) after restricting $h$ to the interval $(0, \pi /(\sigma+2 \kappa))$.

In the rest of this section, we investigate the operator $\mathcal{G}_{n, h, N}$ for $n$-variate analytic functions defined on the multidimensional horizontal strip

$$
S_{d}^{n}:=\left\{z \in \mathbb{C}^{n}:\left|\Im z_{j}\right|<d \text { for all } j=1, \ldots, n\right\} .
$$

Denote by $A_{d}^{n}(\varphi)$ the class of $n$-variate analytic functions $f: S_{d}^{n} \rightarrow \mathbb{C}^{n}$ which satisfy the following condition:

$$
|f(z)| \leq \varphi\left(\left|\Re z_{1}\right|, \ldots,\left|\Re z_{n}\right|\right), \quad z:=\left(z_{1}, \ldots, z_{n}\right) \in S_{d}^{n},
$$

where $\varphi$ is defined after (2.1). For functions from the class $A_{d}^{n}(\varphi)$, we study the operator $\mathcal{G}_{n, h, N}$ in the special case $h:=d / N$ and $\beta=\pi / 2$. In the following theorem, we establish a bound for the error $\left|f(z)-\mathcal{G}_{n, \frac{d}{N}, N}[f](z)\right|$, where $f \in A_{d}^{n}(\varphi)$ and $\beta=\pi / 2$.

THEOREM 2.7. Assume that $f \in A_{d}^{n}(\varphi)$. Then for $z \in S_{d / 4}^{n}$, we have

$$
\begin{aligned}
\left|f(z)-\mathcal{G}_{n, \frac{d}{N}, N}[f](z)\right| & \leq \varphi(\eta(z)) \sum_{m=1}^{n}\left[\left(\frac{2 \sqrt{2}}{\pi}\right)^{m}\right. \\
& \left.\sum_{N_{m} \in P(I)} \prod_{j \in N_{m}}\left|\sin \left(\frac{\pi N z_{j}}{d}\right)\right| \vartheta_{N}\left(\frac{\Im z_{j}}{d}\right) \frac{e^{-\frac{\pi N}{2}\left(1-\frac{2\left|\Im z_{j}\right|}{d}\right)}}{\sqrt{N}}\right],
\end{aligned}
$$

where the functions $\varphi$ and $\eta$ are given in (2.1) and (2.12) (with $h=d / N)$, respectively. The function $\vartheta_{N}$ is defined by

$$
\begin{aligned}
\vartheta_{N}(t) & :=\frac{1}{1-t}\left(\frac{1}{1-e^{-2 \pi N}}+\frac{2 \sqrt{2}}{\pi \sqrt{N}(1+t)}\right) \\
& =\frac{1}{1-t}\left(1+O\left(N^{-1 / 2}\right)\right) \quad \text { as } N \rightarrow \infty .
\end{aligned}
$$

Proof. Let $\mathcal{R}_{j}$ to be the positive oriented rectangle with vertices at $\pm h\left(N+\frac{1}{2}\right)+h N_{h^{-1} z_{j}}+\mathrm{i} d$ and $\pm h\left(N+\frac{1}{2}\right)+h N_{h^{-1} z_{j}}-\mathrm{i}\left(d-\Im z_{j}\right) \quad$ if $\Im z_{j}>0$ where $N_{z_{j}}=\left\lfloor\Re z_{j}+\frac{1}{2}\right\rfloor$ and $h=d / N$. When $\Im z_{j}<0$, the vertices are

$$
\pm h\left(N+\frac{1}{2}\right)+h N_{h^{-1} z_{j}}-\mathrm{i} d \text { and } \pm h\left(N+\frac{1}{2}\right)+h N_{h^{-1} z_{j}}+\mathrm{i}\left(d-\Im z_{j}\right) .
$$


Since $f \in A_{d}^{n}(\varphi)$, it is easy to see that the integral representation in (2.6) is also valid for the special operator $\mathcal{G}_{n, \frac{d}{N}, N}$ with the multivariate rectangle $\prod_{j=1}^{n} \mathcal{R}_{j}$. Applying the triangle inequality for (2.15) after replacing the rectangle $R_{j}$ by $\mathcal{R}_{j}$ with taking $h=\frac{d}{N}$ and $\beta=\frac{\pi}{2}$ and using (2.16), we obtain

$$
\begin{aligned}
\left|f(z)-\mathcal{G}_{n, \frac{d}{N}, N}[f](z)\right| & \\
\leq \frac{1}{(2 \pi)^{m}} \sum_{m=1}^{n} & {\left[\sum_{N_{m} \in P(I)} \prod_{j \in N_{m}}\left|\sin \left(\frac{\pi N z_{j}}{d}\right)\right|\right.} \\
& \left.\quad \times \oint_{\mathcal{R}_{j}}\left|\frac{e^{-N \pi\left(z_{j}-w_{j}\right)^{2} / 2 d^{2}}\left\{\left.f(w)\right|_{w_{l}=z_{l}}\right\}_{l \in M_{m}}}{\sin \left(\frac{\pi N w_{j}}{d}\right)\left(w_{j}-z_{j}\right)}\right|\left|d w_{j}\right|\right] .
\end{aligned}
$$

Since $f \in A_{d}^{n}(\varphi)$, for all points $w$ on the multivariate rectangle $\prod_{j=1}^{n} \mathcal{R}_{j}$, we have

$$
|f(w)| \leq \varphi(\eta(z))
$$

where the function $\eta$ is defined in (2.12). Similarly for all $w \in \prod_{j=1}^{n} \mathcal{R}_{j}$, we obtain that

$$
\left|\left\{\left.f(w)\right|_{w_{l}=z_{l}}\right\}_{l \in M_{m}}\right| \leq \varphi(\eta(z))
$$

for all $M_{m} \subset I$. This is a consequence of the assumption that the function $\varphi$ is nondecreasing for all variables $\left|\Re z_{j}\right|, j=1, \ldots, n$, and $b\left(z_{j}\right)=\left|\Re z_{j}\right|+h(N+1)$. Applying the same technique as in Theorem 2.3 with the use of (2.33) and (2.34), we obtain

$$
\begin{aligned}
& \left|f(z)-\mathcal{G}_{n, \frac{d}{N}, N}[f](z)\right| \\
& \leq \varphi(\eta(z)) \sum_{m=1}^{n}\left[\frac{1}{(2 \pi)^{m}} \sum_{N_{m} \in P(I)} \prod_{j \in N_{m}}\left|\sin \left(\frac{\pi N z_{j}}{d}\right)\right|\right. \\
& \left.\quad \times \oint_{\mathcal{R}_{j}}\left|\frac{e^{\frac{-N \pi\left(z_{j}-w_{j}\right)^{2}}{2 d^{2}}}}{\sin \left(\frac{\pi N w_{j}}{d}\right)\left(w_{j}-z_{j}\right)}\right|\left|d w_{j}\right|\right] .
\end{aligned}
$$

By splitting the contour integral over $\mathcal{R}_{j}$ into four integrals along line segments and transforming the latter into ordinary integrals, one can imitate the estimates in [18, pp. 209-211] to obtain

$$
\oint_{\mathcal{R}_{j}}\left|\frac{e^{\frac{-N \pi\left(z_{j}-w_{j}\right)^{2}}{2 d^{2}}}}{\sin \left(\frac{\pi N w_{j}}{d}\right)\left(w_{j}-z_{j}\right)}\right|\left|d w_{j}\right| \leq 4 \sqrt{2} \vartheta_{N}\left(\frac{\Im z_{j}}{d}\right) \frac{e^{\frac{-\pi N}{2}\left(1-\frac{2\left|\Im z_{j}\right|}{d}\right)}}{\sqrt{N}} .
$$

The proof is completed by combining (2.36) and (2.35).

The bound in (2.30) depends on the growth of the functions $\varphi$ and the behavior of the term

$$
\sum_{m=1}^{n}\left(\frac{2 \sqrt{2}}{\pi}\right)^{m} \sum_{N_{m} \in P(I)} \prod_{j \in N_{m}}\left|\sin \left(\frac{\pi N z_{j}}{d}\right)\right| \vartheta_{N}\left(\frac{\Im z_{j}}{d}\right) \frac{e^{-\frac{\pi N}{2}\left(1-\frac{2\left|\Im z_{j}\right|}{d}\right)}}{\sqrt{N}}
$$


On the real domain, the bound (2.35) will be of exponential order and depends only on the growth of the functions $\varphi$. Indeed, for all $f \in A_{d}^{n}(\varphi)$ and for $x \in \mathbb{R}^{n}$, we have

$$
\left|f(x)-\mathcal{G}_{n, \frac{d}{N}, N}[f](x)\right| \leq \varphi(\eta(x)) \sum_{m=1}^{n}\left(\begin{array}{c}
n \\
m
\end{array}\right)\left(\frac{2 \sqrt{2}}{\pi}\right)^{m} \vartheta_{N}^{m}(0)\left(\frac{e^{-\frac{\pi N}{2}}}{\sqrt{N}}\right)^{m} .
$$

3. Approximation of partial derivatives. In this section, we extend our work in the last section to the approximation of the partial derivatives of any order of a function $f$ of several variables that belongs to the class $E_{\sigma}^{n}(\varphi)$ or $A_{d}^{n}(\varphi)$. Let, here and throughout the paper, $\alpha:=\left(\alpha_{1}, \ldots, \alpha_{n}\right), \alpha_{j} \in \mathbb{N}_{0}, j=1, \ldots, n$ and $D_{z}^{\alpha}:=\frac{\partial^{\alpha_{1}+\ldots+\alpha_{n}}}{\partial z_{1}^{\alpha_{1}} \ldots \partial z_{n}^{\alpha_{n}}}$. We define the partial derivatives of the operator $\mathcal{G}_{n, h, N}$ as

$$
D_{z}^{\alpha} \mathcal{G}_{n, h, N}[f](z):=\sum_{k \in \mathbb{Z}_{N}^{n}(z)} f(k h) D_{z}^{\alpha}\left[\prod_{j=1}^{n} \operatorname{sinc}\left(\pi h^{-1} z_{j}-k_{j} \pi\right) \exp \left(-\frac{\beta\left(z_{j}-k_{j} h\right)^{2}}{N h^{2}}\right)\right] .
$$

In the following result, we show that the approximation error for the partial derivatives of functions from $E_{\sigma}^{n}(\varphi)$ using the operator $D^{\alpha} \mathcal{G}_{n, h, N}$ can be written as an integral of the partial derivatives of the kernel $\mathcal{K}_{z}$ over the multivariate rectangle $\prod_{j=1}^{n} R_{j}$.

Lemma 3.1. Let $f \in E_{\sigma}^{n}(\varphi)$ and $\alpha:=\left(\alpha_{1}, \ldots, \alpha_{n}\right) \in \mathbb{N}_{0}^{n}$. Then we have for all $z \in \mathbb{C}^{n}$,

$$
D_{z}^{\alpha} f(z)-D_{z}^{\alpha} \mathcal{G}_{n, h, N}[f](z)=\frac{1}{(2 \pi \mathrm{i})^{n}} \oint_{R_{n}} \ldots \oint_{R_{1}} D_{z}^{\alpha} \mathcal{K}_{z}(w) f(w) d w_{1} \ldots d w_{n},
$$

where $\mathcal{K}_{z}$ is the kernel function defined in (2.4) and $R_{j}, j=1, \ldots, n$, is the positively oriented rectangle defined above.

Proof. Since $f \in E_{\sigma}^{n}(\varphi)$, the identity (2.6) is valid. Taking the partial derivatives of order $\alpha$ on both sides of (2.6) and observing that differentiation under the integral sign is allowed, we get (3.2).

For all $f \in E_{\sigma}^{n}(\varphi)$, we define the following function $S_{m}$ of several variables $w_{l}, l \in M_{m}$ :

$$
S_{m}\left(\left[w_{l}\right]_{l \in M_{m}}\right):=\oint_{\prod_{j \in N_{m}} R_{j}} \frac{f(w) \prod_{j \in N_{m}} \partial_{z_{j}}^{\delta_{j}}\left\{\exp \left(-\beta\left(z_{j}-w_{j}\right)^{2} / N h^{2}\right)\right\}}{\prod_{j \in N_{m}}\left(w_{j}-z_{j}\right)^{\gamma_{j}+1}} \prod_{j \in N_{m}} d w_{j},
$$

such that $z_{j}$ lies inside the rectangle $R_{j}$ and $\delta_{j} \in \mathbb{N}_{0}, j \in N_{m}$. Here $\partial_{z_{j}}^{\delta_{j}} f$ denotes the partial derivatives of order $\delta_{j}$ with respect to $z_{j}, j=1, \ldots, n$.

The double factorial of a number $n$, denoted by $n !$ !, is the product of all the integers from 1 up to $n$ that have the same parity (odd or even) as $n$. That is,

$$
n ! !=\prod_{k=0}^{\left\lceil\frac{n}{2}\right\rceil-1}(n-2 k)
$$

where $\lceil x\rceil$ denotes the least integer greater than or equal to $x$. The following lemma is devoted to estimates of the integral on the right-hand side of (3.3), and it will be used in the proof of the main results of this section. 
Lemma 3.2. Assume that $f \in E_{\sigma}^{n}(\varphi)$. Let $\gamma_{N_{m}}=\left(\gamma_{1}, \ldots, \gamma_{n}\right)$ be given such that $\gamma_{j}=0$ if $j \notin N_{m}, \nu_{j} \in \mathbb{N}_{0}$, for all $j=1, \ldots, n$, and $\delta_{j} \in \mathbb{N}_{0}, j \in N_{m}$. Then, we have

$$
\begin{aligned}
& \left|S_{m}\left(\left[w_{l}\right]_{l \in M_{m}}\right)\right| \\
& \leq(2 \pi)^{m} \varphi(\eta(z)) \sum_{\nu_{N_{m}} \leq \gamma_{N_{m}}} \frac{\prod_{j \in N_{m}} e^{\sigma\left(\left|\Im z_{j}\right|+h\right)}\left(\frac{\sqrt{\beta}}{\sqrt{N} h}\right)^{\delta_{j}+\gamma_{j}-\nu_{j}} \mid \mathcal{H}_{\delta_{j}+\gamma_{j}-\nu_{j} \mid} \prod_{l \in M_{m}} e^{\sigma\left|\Im w_{l}\right|}}{\prod_{j \in N_{m}} h^{\nu_{j}}\left(\gamma_{j}-\nu_{j}\right) !},
\end{aligned}
$$

where $S_{m}$ and $\eta$ are defined by (3.3) and (2.12), respectively. The constant $\mathcal{H}_{n}$ is the Hermite number

$$
\mathcal{H}_{n}:= \begin{cases}0, & \text { if } n \text { is odd, } \\ (-1)^{\frac{n}{2}} 2^{\frac{n}{2}}(n-1) ! !, & \text { if } n \text { is even, }\end{cases}
$$

where $n ! !$ is the double factorial of $n$. The sum in (3.4) is taken over all multiindices $\nu_{N_{m}} \leq \alpha_{N_{m}}$, i.e., $\nu_{j} \leq \alpha_{j}$, for all $j \in N_{m}$. Here $\nu_{N_{m}}=\left(\nu_{1}, \ldots, \nu_{n}\right)$ such that $\nu_{j}=0$ if $j \notin N_{m}$ and $\nu_{j} \in \mathbb{N}_{0}$, for all $j=1, \ldots, n$.

Proof. Applying CauchyâĂŹs integral formula, cf., e.g., [17, p. 18], for the right-hand side of (3.3), we obtain

$$
\begin{aligned}
& S_{m}\left(\left[w_{l}\right]_{l \in M_{m}}\right) \\
& =\frac{(2 \pi \mathrm{i})^{m}}{\prod_{j \in N_{m}} \gamma_{j} !}\left\{D_{w}^{\gamma_{N m}}\left(f(w) \prod_{j \in N_{m}} \partial_{z_{j}}^{\delta_{j}}\left\{\exp \left(-\beta\left(z_{j}-w_{j}\right)^{2} / N h^{2}\right)\right\}\right)\right\},
\end{aligned},
$$

where $D_{w}^{\alpha}$ is defined as above. The multivariate version of the Leibniz rule is given by, cf., e.g., [8, p. 13],

$$
D_{w}^{\alpha}(f(w) g(w))=\sum_{\nu \leq \alpha} \frac{\prod_{j=1}^{n} \alpha_{j} !}{\prod_{j=1}^{n} \nu_{j} !\left(\alpha_{j}-\nu_{j}\right) !} D_{w}^{\nu} f(w) D_{w}^{\alpha-\nu} g(w),
$$

where $f, g$ are analytic functions of several variables, $w:=\left(w_{1}, \ldots, w_{n}\right), \alpha:=\left(\alpha_{1}, \ldots, \alpha_{n}\right)$, and $\nu:=\left(\nu_{1}, \ldots, \nu_{n}\right)$. The sum in (3.7) is taken over all multiindices $\nu:=\left(\nu_{1}, \ldots, \nu_{n}\right)$, and here $\nu \leq \alpha$ means that $\nu_{j} \leq \alpha_{j}(j=1, \ldots, n)$. Applying formula (3.7) for the right-hand side of (3.6), we obtain

$$
\begin{aligned}
& S_{N_{m}}\left(\left[w_{l}\right]_{l \in M_{m}}\right) \\
& =(2 \pi \mathrm{i})^{m} \sum_{\nu_{N_{m}} \leq \gamma_{N_{m}}}\left[\frac{1}{\prod_{j \in N_{m}} \nu_{j} !\left(\gamma_{j}-\nu_{j}\right) !}\right. \\
& \left.\times\left\{D_{w}^{\nu_{N_{m}}} f(w) \prod_{j \in N_{m}} \partial_{z_{j}, w_{j}}^{\delta_{j}, \gamma_{j}-\nu_{j}}\left\{e^{\frac{-\beta\left(z_{j}-w_{j}\right)^{2}}{N h^{2}}}\right\}\right\}_{\substack{w_{j}=z_{j} \\
j \in N_{m}}}\right],
\end{aligned}
$$

where $\nu_{N_{m}}$ is defined above and $\partial_{z_{i}, w_{j}}^{\delta_{i}, \gamma_{j}} f$ are the higher-order partial and mixed derivatives, i.e., $\partial_{z_{i}, w_{j}}^{\delta_{i}, \gamma_{j}} f:=\partial_{z_{i}}^{\delta_{i}} \partial_{w_{j}}^{\gamma_{j}} f$. From [7], we have

$$
\partial_{\zeta, \xi}^{\tau_{1}, \tau_{2}}\left\{e^{\frac{-\beta(\zeta-\xi)^{2}}{N h^{2}}}\right\}=(-1)^{\tau_{1}+\tau_{2}}\left(\frac{\sqrt{\beta}}{\sqrt{N} h}\right)^{\tau_{1}+\tau_{2}} H_{\tau_{1}+\tau_{2}}\left(\frac{\sqrt{\beta}(\zeta-\xi)}{\sqrt{N} h}\right) e^{\frac{-\beta(\zeta-\xi)^{2}}{N h^{2}}}
$$


where $\zeta, \xi \in \mathbb{C}, \tau_{1}, \tau_{2}$ are integer numbers and $H_{n}$ is the $n$th degree Hermite polynomial defined by $H_{n}(x):=(-1)^{n} e^{x^{2}} \frac{d^{n}}{d x^{n}} e^{-x^{2}}$. Using (3.9), we have

$$
\left\{\partial_{\zeta, \xi}^{\tau_{1}, \tau_{2}}\left\{e^{\frac{-\beta(\zeta-\xi)^{2}}{N h^{2}}}\right\}\right\}_{\xi=\zeta}=(-1)^{\tau_{1}+\tau_{2}}\left(\frac{\sqrt{\beta}}{\sqrt{N h}}\right)^{\tau_{1}+\tau_{2}} \mathcal{H}_{\tau_{1}+\tau_{2}}
$$

where the last step results from the monomial representation of the $n$th degree Hermite polynomial and $\mathcal{H}_{\tau_{1}+\tau_{2}}$ is the Hermite number given in (3.5). The derivatives $\left\{D^{\nu_{N_{m}}} f(w)\right\} \underset{\substack{w_{j}=z_{j} \\ j \in N_{m}}}{\substack{w_{j} \\ \text {, }}}$ can be represented using CauchyâĂŹs integral formula [17, p. 18] as follows:

$$
\left\{D_{w}^{\nu_{N_{m}}} f(w)\right\}_{w_{j} z_{j}, j \in N_{m}}=\frac{\prod_{j \in N_{m}} \nu_{j} !}{(2 \pi \mathrm{i})^{m}} \oint_{\Omega_{m}\left(z_{j}, h\right)} \frac{f(w)}{\prod_{j \in N_{m}}\left(w_{j}-z_{j}\right)^{\nu_{j}+1}} \prod_{j \in N_{m}} d w_{j},
$$

where $\Omega_{m}\left(z_{j}, h\right):=\left\{z_{j} \in \mathbb{C}:\left|w_{j}-z_{j}\right|=h, j \in N_{m}\right\}$ is the $m$-dimensional sphere. Since $f \in E_{\sigma}^{n}(\varphi)$, inequality (2.1) holds. Applying Cauchy estimates to (3.11) and using inequality (2.1), we obtain

$$
\left\{\left|D_{w}^{\nu_{N m}} f(w)\right|\right\}_{w_{j}=z_{j}, j \in N_{m}} \leq \frac{\prod_{j \in N_{m}} \nu_{j} !}{\prod_{j \in N_{m}} h^{\nu_{j}}} \varphi(\eta(z)) \prod_{j \in N_{m}} e^{\sigma\left(\left|\Im z_{j}\right|+h\right)} \prod_{l \in M_{m}} e^{\sigma\left|\Im w_{l}\right|},
$$

where $\eta(z)$ is defined in (2.12). Combining (3.12) and (3.10) with (3.8) implies (3.4).

Lemma 3.3. Let $\alpha:=\left(\alpha_{1}, \ldots, \alpha_{n}\right), \mu:=\left(\mu_{1}, \ldots, \mu_{n}\right)$, and $v:=\left(v_{1}, \ldots, v_{n}\right)$ in $\mathbb{N}_{0}^{n}$. The partial derivatives of the kernel $\mathcal{K}_{z}$ can be written as

$$
\begin{array}{r}
D_{z}^{\alpha} \mathcal{K}_{z}(w) \\
=\sum_{m=1}^{n}(-1)^{m+1} \sum_{N_{m} \in P(I)} \sum_{\mu \leq \alpha} \sum_{\mu+v \leq \alpha}\left[C_{\alpha, \mu, v} \prod_{j \in N_{m}}\left(\frac{\pi}{h}\right)^{\mu_{j}} \frac{\sin \left(\pi h^{-1} z_{j}+\frac{\pi \mu_{j}}{2}\right)}{\sin \left(\pi h^{-1} w_{j}\right)}\right. \\
\left.\times \prod_{\tau=1}^{n} \frac{\partial_{z_{\tau}}^{v_{\tau}}\left\{\exp \left(\frac{-\beta\left(z_{\tau}-w_{\tau}\right)^{2}}{N h^{2}}\right)\right\}}{\left(w_{\tau}-z_{\tau}\right)^{\alpha_{\tau}-\mu_{\tau}-v_{\tau}+1}}\right],
\end{array}
$$

where the symbol $D_{z}^{\alpha}$ is defined above, the kernel $\mathcal{K}_{z}$ is given in (2.4), and the constants $C_{\alpha, \mu, v}$ are defined as

$$
C_{\alpha, \mu, v}:=\prod_{j=1}^{n} \frac{\alpha_{j} !}{\mu_{j} ! v_{j} !}
$$

The sum $\sum_{\mu \leq \alpha}$ is taken over all multiindices $\mu \leq \alpha$, i.e., $\mu_{j} \leq \alpha_{j}$, for all $j=1, \ldots, n$.

Proof. Taking the partial derivative of order $\alpha$ on both sides of (2.4) and using (2.10), we obtain

$$
D_{z}^{\alpha} \mathcal{K}_{z}(w)=\sum_{m=1}^{n}(-1)^{m+1} \sum_{N_{m} \in P(I)} D_{z}^{\alpha}\left[\prod_{j \in N_{m}} \frac{\sin \left(\pi h^{-1} z_{j}\right)}{\sin \left(\pi h^{-1} w_{j}\right)} \prod_{\tau=1}^{n} \frac{e^{-\beta\left(z_{\tau}-w_{\tau}\right)^{2} / N h^{2}}}{\left(w_{\tau}-z_{\tau}\right)}\right]
$$


Applying the multivariate version of the Leibniz rule (3.7) for the right-hand side of (3.15) with $f(z):=\prod_{j \in N_{m}} \frac{\sin \left(\pi h^{-1} z_{j}\right)}{\sin \left(\pi h^{-1} w_{j}\right)}$ and $g(z):=\prod_{\tau=1}^{n} \frac{e^{-\beta\left(z_{\tau}-w_{\tau}\right)^{2} / N h^{2}}}{\left(w_{\tau}-z_{\tau}\right)}$, we obtain

$$
\begin{aligned}
& D_{z}^{\alpha} \mathcal{K}_{z}(w) \\
& =\sum_{m=1}^{n}(-1)^{m+1} \sum_{N_{m} \in P(I)} \sum_{\mu \leq \alpha}\left[\left(\begin{array}{l}
\alpha \\
\mu
\end{array}\right) \prod_{j \in N_{m}}\left(\frac{\pi}{h}\right)^{\mu_{j}} \frac{\sin \left(\pi h^{-1} z_{j}+\frac{\pi \mu_{j}}{2}\right)}{\sin \left(\pi h^{-1} w_{j}\right)}\right. \\
& \left.\times D_{z}^{\alpha-\mu}\left[\prod_{\tau=1}^{n} \frac{e^{-\beta\left(z_{\tau}-w_{\tau}\right)^{2} / N h^{2}}}{\left(w_{\tau}-z_{\tau}\right)}\right]\right] .
\end{aligned}
$$

Applying the multivariate version of the Leibniz rule (3.7) again with

$$
f(z):=\prod_{\tau=1}^{n} e^{-\beta\left(z_{\tau}-w_{\tau}\right)^{2} / N h^{2}} \quad \text { and } \quad g(z):=\prod_{\tau=1}^{n} \frac{1}{w_{\tau}-z_{\tau}},
$$

we get (3.13).

In the following theorem, we extend the bound for the error $\left|f(z)-\mathcal{G}_{n, h, N}[f](z)\right|$ given in Theorem 2.3 to a bound for the error $\left|D_{z}^{\alpha} f(z)-D_{z}^{\alpha} \mathcal{G}_{n, h, N}[f](z)\right|$, where $f \in E_{\sigma}^{n}(\varphi)$.

THEOREM 3.4. Let $f \in E_{\sigma}^{n}(\varphi)$ and $\alpha:=\left(\alpha_{1}, \ldots, \alpha_{n}\right) \in \mathbb{N}_{0}^{n}$. Then, we have for all $z \in \mathbb{C}^{n}$ with $\left|\Im z_{j}\right|<N, j=1, \ldots, n$, that

$$
\left|D_{z}^{\alpha} f(z)-D_{z}^{\alpha} \mathcal{G}_{n, h, N}[f](z)\right| \leq 2 \varphi(\eta(z)) \mathbb{B}_{h, N, \alpha}(\Im z) \frac{e^{-\beta N}}{\sqrt{\pi \beta N}},
$$

where $\eta(z)$ is defined in $(2.12)$ and $\mathbb{B}_{h, N, \alpha}$ is given by

$$
\begin{aligned}
& \mathbb{B}_{h, N, \alpha}(\Im z) \\
& =\prod_{\tau=1}^{n} e^{\pi h^{-1}\left|\Im z_{\tau}\right|} \sum_{\mu \leq \alpha} \sum_{\mu+v \leq \alpha} C_{\alpha, \mu, v}\left[\sum_{m=1}^{n}\left(\frac{2 e^{-\beta N}}{\sqrt{\pi \beta N}}\right)^{m-1}\right. \\
& \left.\quad \times \sum_{N_{m} \in P(I)} \prod_{l \in M_{m}} e^{\sigma h} L_{h, N}\left(v_{l}, \gamma_{l}\right) \prod_{j \in N_{m}}\left(\frac{\pi}{h}\right)^{\mu_{j}} \frac{M_{h, N}\left(v_{j}\right) \theta_{N}\left(\frac{\Im z_{j}}{h}\right)}{N^{\alpha_{j}-\mu_{j}-v_{j}}}\right] .
\end{aligned}
$$

Here $\theta_{N}, C_{\alpha, \mu, v}$ are defined in (2.14), (3.14), respectively, and

$$
L_{h, N}(v, \gamma):=\sum_{\nu_{M_{m}} \leq \gamma_{M_{m}}} \frac{\left(\frac{\sqrt{\beta}}{\sqrt{N} h}\right)^{v-\gamma-\nu}\left|\mathcal{H}_{v+\gamma-\nu}\right|}{h^{\nu}(\alpha-\nu) !}=O\left(N^{-v / 2}\right) \quad \text { as } N \rightarrow \infty
$$

where $\gamma_{M_{m}}:=\alpha_{M_{m}}-\mu_{M_{m}}-v_{M_{m}}$ such that $\gamma_{M_{m}}=0$ if $l \notin M_{m}$ and

$$
M_{h, N}(v):=\frac{\beta^{\frac{v}{2}}}{h^{v}} \sum_{m=0}^{\lfloor v / 2\rfloor} \frac{v !(6 \sqrt{\beta})^{v-2 m}}{m !(v-2 m) ! N^{m}}=\left(6 \beta h^{-1}\right)^{v}+O\left(N^{-1}\right) \quad \text { as } N \rightarrow \infty .
$$

The constant $\mathcal{H}_{\tau}$ is defined in (3.5). 
Proof. Since $f \in E_{\sigma}^{n}(\varphi)$, equality (3.2) holds. Using (3.13), the integral in (3.2) can be represented in the form

$$
\begin{aligned}
& D_{z}^{\alpha} f(z)-D_{z}^{\alpha} \mathcal{G}_{n, h, N}[f](z) \\
& =\frac{1}{(2 \pi \mathrm{i})^{n}} \sum_{m=1}^{n}(-1)^{m+1} \sum_{\mu \leq \alpha} \sum_{\mu+v \leq \alpha}\left[C_{\alpha, \mu, v} \prod_{j \in N_{m}}\left(\frac{\pi}{h}\right)^{\mu_{j}} \sin \left(\frac{\pi z_{j}}{h}+\frac{\pi \mu_{j}}{2}\right)\right. \\
& \left.\quad \times \oint_{R_{n}} \ldots \oint_{R_{1}} \frac{f(w) \prod_{\tau=1}^{n} \partial_{z_{\tau}}^{v_{\tau}}\left\{\exp \left(\frac{-\beta\left(z_{\tau}-w_{\tau}\right)^{2}}{N h^{2}}\right)\right\}}{\prod_{j \in N_{m}} \sin \left(\pi h^{-1} w_{j}\right) \prod_{\tau=1}^{n}\left(w_{\tau}-z_{\tau}\right)^{\alpha_{\tau}-\mu_{\tau}-v_{\tau}+1}} d w_{1} \ldots d w_{n}\right] .
\end{aligned}
$$

Using (3.3), replacing the sets $N_{m}$ by $M_{m}$, we obtain

$$
\begin{aligned}
& D_{z}^{\alpha} f(z)-D_{z}^{\alpha} \mathcal{G}_{n, h, N}[f](z) \\
& =\frac{1}{(2 \pi \mathrm{i})^{n}} \sum_{m=1}^{n}(-1)^{m+1} \sum_{N_{m} \in P(I)} \sum_{\mu \leq \alpha} \sum_{\mu+v \leq \alpha}\left[C_{\alpha, \mu, v} \prod_{j \in N_{m}}\left(\frac{\pi}{h}\right)^{\mu_{j}} \sin \left(\frac{\pi z_{j}}{h}+\frac{\pi \mu_{j}}{2}\right)\right. \\
& \left.\times \oint_{\prod_{\tau \in N_{m}} R_{\tau}} \frac{S_{m}\left(\left[w_{l}\right]_{l \in N_{m}}\right) \prod_{\tau \in N_{m}} \partial_{z_{\tau}}^{v_{\tau}}\left\{\exp \left(\frac{-\beta\left(z_{\tau}-w_{\tau}\right)^{2}}{N h^{2}}\right)\right\}}{\prod_{\tau \in N_{m}} \sin \left(\pi h^{-1} w_{\tau}\right)\left(w_{\tau}-z_{\tau}\right)^{\alpha_{\tau}-\mu_{\tau}-v_{\tau}+1}} \prod_{\tau \in N_{m}} d w_{\tau}\right],
\end{aligned}
$$

where the function $S_{M_{m}}$ is defined by (3.3) and the constants $C_{\alpha, \mu, v}$ are given in (3.14). Since $f \in E_{\sigma}^{n}(\varphi)$, the estimate in (2.18) holds for all points in the multivariate rectangle $\prod_{\tau=1}^{n} R_{\tau}$. In the following step, we use the standard estimate

$$
\left|\sin \left(\pi h^{-1} z\right)\right| \leq e^{\pi h^{-1}|\Im z|}, \quad z \in \mathbb{C},
$$

and Asharabi-Prestin's estimate, cf. [7, Eqs. (3.8)-(3.9)],

$$
\left|\partial_{z_{\tau}}^{v_{\tau}} e^{\frac{-\beta\left(z_{\tau}-w_{\tau}\right)^{2}}{N h^{2}}}\right| \leq\left(\frac{\sqrt{\beta}}{\sqrt{N} h}\right)^{v_{\tau}} \sum_{m=0}^{\left\lfloor\frac{v_{\tau}}{2}\right\rfloor} \frac{v_{\tau} !(6 \sqrt{\beta})^{v_{\tau}-2 m}}{m !\left(v_{\tau}-2 m\right) !} N^{\frac{v_{\tau}}{2}-m}\left|e^{\frac{-\beta\left(z_{\tau}-w_{\tau}\right)^{2}}{N h^{2}}}\right|
$$

for all $w_{\tau} \in R_{\tau}$. Applying the triangle inequality for (3.22) and using the estimates (2.18), (3.4), (3.23), and (3.24), we obtain

$$
\begin{aligned}
\left|D_{z}^{\alpha} f(z)-D_{z}^{\alpha} \mathcal{G}_{n, h, N}[f](z)\right| & \\
\leq \varphi(\eta(z)) \sum_{m=1}^{n} \frac{1}{(2 \pi)^{m}} & \sum_{N_{m} \in P(I)} \sum_{\mu \leq \alpha} \sum_{\mu+v \leq \alpha}\left[C_{\alpha, \mu, v} \prod_{l \in M_{m}} e^{\sigma\left(\left|\Im z_{l}\right|+h\right)}\right. \\
& \times L_{h, N}\left(v_{l}, \gamma_{l}\right) \prod_{j \in N_{m}}\left(\frac{\pi}{h}\right)^{\mu_{j}} e^{\pi h^{-1}\left|\Im z_{j}\right|} M_{h, N}\left(v_{j}\right) \\
& \left.\times \oint_{R_{j}}\left|\frac{\exp \left(\sigma\left|\Im w_{j}\right|-\frac{\beta\left(z_{j}-w_{j}\right)^{2}}{N h^{2}}\right)}{\sin \left(\pi h^{-1} w_{j}\right)\left(w_{j}-z_{j}\right)^{\alpha_{j}-\mu_{j}-v_{j}+1}}\right|\left|d w_{j}\right|\right],
\end{aligned}
$$


where the functions $L_{h, N}$ and $M_{h, N}$ are defined in (3.19) and (3.20), respectively. The integral in (3.25) can be estimated according to Asharabi and Prestin in [7, p. 1429] as follows:

$$
\oint_{R_{\tau}}\left|\frac{\exp \left(\sigma\left|\Im w_{\tau}\right|-\frac{\beta\left(z_{\tau}-w_{\tau}\right)^{2}}{N h^{2}}\right)}{\sin \left(\pi h^{-1} w_{\tau}\right)\left(w_{\tau}-z_{\tau}\right)^{l_{\tau}+1}}\right|\left|d w_{\tau}\right| \leq 4 \pi \theta_{N}\left(h^{-1} \Im z_{\tau}\right) \frac{e^{-\beta N}}{N^{\ell_{\tau}} \sqrt{\pi \beta N}},
$$

where $\ell_{\tau} \in \mathbb{N}_{0}$. The rest of the proof can be established by combining (3.26) and (3.25).

As we have done in Section 2, we introduce two useful special cases of Theorem 3.4. The proofs will be omitted because they are similar to those of Corollary 2.5 and Corollary 2.6, respectively.

COROLlaRy 3.5. Let $f \in B_{\sigma}^{\infty}\left(\mathbb{R}^{n}\right)$ and $\alpha \in \mathbb{N}_{0}^{n}$ be given. Then we have for all $z \in \mathbb{C}^{n}$ with $\left|\Im z_{\tau}\right|<N, \tau=1, \ldots, n$, that

$$
\left|D_{z}^{\alpha} f(z)-D_{z}^{\alpha} \mathcal{G}_{n, h, N}[f](z)\right| \leq 2\|f\|_{\infty} \mathbb{B}_{h, N, \alpha}(\Im z) \frac{e^{-\beta N}}{\sqrt{\pi \beta N}}
$$

where the function $\mathbb{B}_{h, N, \alpha}$ is given in (3.18) and the norm $\|f\|_{\infty}$ is given in (1.1).

COROLlary 3.6. Let $f$ be an entire function satisfying the exponential growth condition (2.27). Then we have for all $z \in \mathbb{C}^{n}$ with $\left|\Im z_{\tau}\right|<N, \tau=1, \ldots, n$, that

$$
\left|D_{z}^{\alpha} f(z)-D_{z}^{\alpha} \mathcal{G}_{n, h, N}[f](z)\right| \leq 2 M e^{\kappa\left(h+\sum_{j=1}^{n}\left|\Re z_{j}\right|\right)} \mathbb{B}_{h, N, \alpha}(\Im z) \frac{e^{-(\beta-\kappa h) N}}{\sqrt{\pi \beta N}},
$$

where $h \in(0, \pi /(\sigma+2 \kappa))$ and $\sigma, \kappa$ are non-negative numbers that satisfy $\sigma+\kappa>0$. The function $\mathbb{B}_{h, N, \alpha}$ is given in (3.18).

The last result in this section is devoted to estimating the error $\left|D_{z}^{\alpha} f(z)-D_{z}^{\alpha} \mathcal{G}_{n, h, N}[f](z)\right|$ when the function $f$ belongs to the space $A_{d}^{n}(\varphi)$ (with $h:=\frac{d}{N}$ and $\beta:=\frac{\pi}{2}$ ).

THEOREM 3.7. Let $f \in A_{d}^{n}(\varphi)$. Then, for $z \in S_{d / 4}^{n}$ we have

$$
\left|D_{z}^{\alpha} f(z)-D_{z}^{\alpha} \mathcal{G}_{n, \frac{d}{N}, N}[f](z)\right|
$$

$$
\begin{aligned}
& \leq 2 \sqrt{2} \varphi(\eta(z)) \sum_{\mu \leq \alpha} \sum_{\mu+v \leq \alpha} C_{\alpha, \mu, v}\left[\sum_{m=1}^{n}(2 \sqrt{2})^{m-1} \sum_{N_{m} \in P(I)} \prod_{\in \in M_{m}} L_{\frac{d}{N}, N}\left(v_{l}, \gamma_{l}\right)\right. \\
& \left.\times \prod_{j \in N_{m}} \frac{\left(\frac{N \pi}{d}\right)^{\mu_{j}} e^{\frac{N \pi\left|\Im z_{j}\right|}{d}} M_{\frac{d}{N}, N}\left(v_{j}\right)}{\pi N^{\alpha_{j}-\mu_{j}-v_{j}} \sqrt{N}} \vartheta_{N}\left(\frac{\Im z_{j}}{d}\right) e^{-\frac{\pi N}{2}\left(1-\frac{2\left|\Im z_{j}\right|}{d}\right)}\right],
\end{aligned}
$$

where the functions $\eta, L_{\frac{d}{N}, N}, M_{\frac{d}{N}, N}$, and $\vartheta_{N}$ are defined in (2.12), (3.19), (3.20), and (2.31), respectively. The constants $C_{\alpha, \mu, v}$ are given in (3.14).

Proof. As in the proof of Theorem 2.7, let $\mathcal{R}_{j}$ be the positive oriented rectangle with vertices at $\pm h\left(N+\frac{1}{2}\right)+h N_{h^{-1} z_{j}}+\mathrm{i} d$ and $\pm h\left(N+\frac{1}{2}\right)+h N_{h^{-1} z_{j}}-\mathrm{i} h\left(d-\Im z_{j}\right)$, where $N_{z_{j}}=\left\lfloor\Re z_{j}+\frac{1}{2}\right\rfloor$ and $h=d / N$. Since $f \in A_{d}^{n}(\varphi)$, it is easy to see that Lemma 3.1 is also valid for the special operator $\mathcal{G}_{n, \frac{d}{N}, N}$ with the multivariate rectangle $\prod_{j=1}^{n} \mathcal{R}_{j}$. Combining (3.2), 
(3.3), and (3.13) with $h:=\frac{d}{N}, \beta:=\frac{\pi}{2}, R_{\tau}:=\mathcal{R}_{\tau}$, we obtain

$$
\begin{aligned}
& D_{z}^{\alpha} f(z)-D_{z}^{\alpha} \mathcal{G}_{n, \frac{d}{N}, N}[f](z) \\
& =\frac{1}{(2 \pi \mathrm{i})^{n}} \sum_{m=1}^{n}(-1)^{m+1} \sum_{N_{m} \in P(I)} \sum_{\mu \leq \alpha} \sum_{\mu+v \leq \alpha}\left[C_{\alpha, \mu, v} \prod_{j \in N_{m}}\left(\frac{\pi}{h}\right)^{\mu_{j}} \sin \left(\frac{\pi z_{j}}{h}+\frac{\pi \mu_{j}}{2}\right)\right.
\end{aligned}
$$

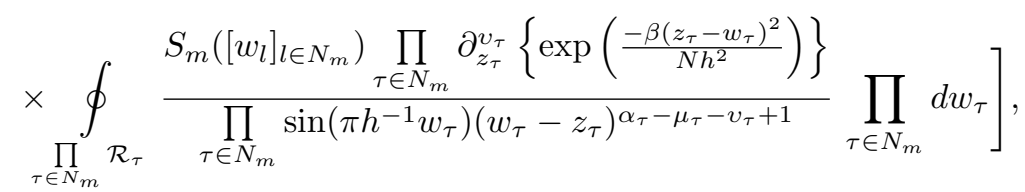

where the function $S_{M_{m}}$ is defined in (3.3) and the constants $C_{\alpha, \mu, v}$ are given in (3.14). Applying the triangle inequality to (3.30) and using the estimates (3.23), (3.24), and (3.4), this implies

$$
\begin{aligned}
& \left|D_{z}^{\alpha} f(z)-D_{z}^{\alpha} \mathcal{G}_{n, \frac{d}{N}, N}[f](z)\right| \\
& \begin{aligned}
\leq \varphi(\eta(z)) \sum_{m=1}^{n} \frac{1}{(2 \pi)^{m}} & \sum_{N_{m} \in P(I)} \sum_{\mu \leq \alpha \mu+v \leq \alpha} \sum_{\mu}\left[C_{\alpha, \mu, v}\right. \\
& \times \prod_{l \in M_{m}} L_{\frac{d}{N}, N}\left(v_{l}, \gamma_{l}\right) \prod_{j \in N_{m}}\left(\frac{\pi N}{d}\right)^{\mu_{j}} e^{\frac{\pi N\left|\Im z_{j}\right|}{d}} M_{\frac{d}{N}, N}\left(v_{j}\right) \\
& \left.\times \oint_{\mathcal{R}_{j}}\left|\frac{e^{\frac{-N \pi\left(z_{j}-w_{j}\right)^{2}}{2 d^{2}}} \mid}{\sin \left(\frac{\pi N w_{j}}{d}\right)\left(w_{j}-z_{j}\right)^{\alpha_{j}-\mu_{j}-v_{j}+1}}\right| d w_{j} \mid\right],
\end{aligned}
\end{aligned}
$$

where the functions $\eta, L_{\frac{d}{N}, N}$, and $M_{\frac{d}{N}, N}$ are defined in (2.12), (3.19), and (3.20), respectively. The integral in (3.25) can be estimated according to Asharabi and Prestin in [7, p. 1432] as

$$
\begin{aligned}
\oint_{\mathcal{R}_{\tau}}\left|\frac{e^{\frac{-N \pi\left(z_{\tau}-w_{\tau}\right)^{2}}{2 d^{2}}}}{\sin \left(\frac{\pi N w_{\tau}}{d}\right)\left(w_{\tau}-z_{\tau}\right)^{\ell_{\tau}+1}}\right|\left|d w_{\tau}\right| \\
\quad \leq 4 \sqrt{2} \vartheta_{N}\left(\frac{\Im z_{\tau}}{d}\right) \frac{\exp \left(-(\pi N / 2)\left(1-\left(2\left|\Im z_{\tau}\right| / d\right)\right)\right)}{N^{\ell_{\tau}} \sqrt{N}},
\end{aligned}
$$

where $\ell_{\tau} \in \mathbb{N}_{0}$ and the function $\vartheta_{N}$ is given in (2.31). The proof of (3.29) is completed by combining (3.32) and (3.31).

In the following result, we state a special case of Theorem 3.7 on the real domain.

COROLlary 3.8. Let $f \in A_{d}^{n}(\varphi)$. Then, for $x \in \mathbb{R}^{n}$ we have

$$
\left|D_{x}^{\alpha} f(x)-D_{x}^{\alpha} \mathcal{G}_{n, \frac{d}{N}, N}[f](x)\right| \leq 2 \sqrt{2} \varphi(\eta(x)) \mathfrak{B}_{d, N, \alpha} \frac{\mathrm{e}^{-\pi N / 2}}{\sqrt{N}},
$$

where $\eta$ is defined by (2.12). The constant $\mathfrak{B}_{d, N, \alpha}$ is defined as

$$
\begin{aligned}
\mathfrak{B}_{d, N, \alpha}=\sum_{\mu \leq \alpha} \sum_{\mu+v \leq \alpha} & {\left[C_{\alpha, \mu, v} \sum_{m=1}^{n}(2 \sqrt{2})^{m-1}\left(\frac{\mathrm{e}^{-\pi N / 2}}{\sqrt{N}}\right)^{m-1} \vartheta_{N}^{m}(0)\right.} \\
& \left.\times \sum_{N_{m} \in P(I)} \prod_{l \in M_{m}} L_{\frac{d}{N}, N}\left(v_{l}, \gamma_{l}\right) \prod_{j \in N_{m}} \frac{\left(\frac{N \pi}{d}\right)^{\mu_{j}} M_{\frac{d}{N}, N}\left(v_{j}\right)}{\pi N^{\alpha_{j}-\mu_{j}-v_{j}}}\right],
\end{aligned}
$$




\section{ETNA}

Kent State University and

Johann Radon Institute (RICAM)

where $\vartheta_{N}$ is given by (2.31).

4. Numerical illustrations. In this section, we introduce three illustrative examples. The function in the first example is chosen from the space $B_{\sigma}^{\infty}(\mathbb{R})$ and in the second example from the space $E_{\sigma}^{2}(\varphi)$, while in the last example $f \in A_{d}^{3}(\varphi)$. We summarize the approximations results in tables and illustrate the absolute and relative errors by figures. Denote by $T_{n, h, N}^{\alpha}$ the absolute error, i.e.,

$$
T_{n, h, N}^{\alpha}[f](x):=D_{x}^{\alpha} f(x)-D_{x}^{\alpha} \mathcal{G}_{n, h, N}[f](x), \quad x \in \mathbb{R}^{n}, \alpha \in \mathbb{N}_{0}^{n},
$$

and by $\mathfrak{T}_{n, h, N}^{\alpha}$ the relative error, i.e.,

$$
\mathfrak{T}_{n, h, N}^{\alpha}[f](x):=\left(D_{x}^{\alpha} f(x)-D_{x}^{\alpha} \mathcal{G}_{n, h, N}[f](x)\right) / D_{x}^{\alpha} f(x), \quad x \in \mathbb{R}^{n}, \alpha \in \mathbb{N}_{0}^{n} .
$$

This notation will be used in the following examples.

EXAMPLE 4.1. The function $f(z)=\operatorname{sinc}\left(\sqrt{1+z^{2}}\right), z \in \mathbb{C}$, belongs to the Bernstein space $B_{1}^{\infty}(\mathbb{R})$. We apply Corollary 3.5 with $n=1, N=15, h=1 / 2$, and $\alpha=0,1$. The function $\varphi$ is chosen to be the constant function $\varphi:=\|f\|_{\infty}=1$, and the bound in (3.17) will be uniform on the real domain. Let $\mathcal{B}_{h, N, \alpha}$ be the uniform bound on $\mathbb{R}$, i.e.,

$$
\mathcal{B}_{h, N}^{\alpha}:=2\|f\|_{\infty} \mathbb{B}_{h, N, \alpha}(0) \frac{e^{-\beta N}}{\sqrt{\pi \beta N}},
$$

where $\alpha \in \mathbb{N}_{0}$ and $\mathbb{B}_{h, N, \alpha}$ are defined in (3.18). Table 4.1 and Figures 4.1 and 4.2 summarize the approximations of the function $f$ and its first derivative on the interval $I=[0,10]$.

TABLE 4.1

Approximation of function $f$ and its first derivative.

Absolute error for $D^{\alpha} \mathcal{G}_{n, h, N}$ with $n=1, N=15$ and $h=1 / 2$

\begin{tabular}{c|c||c|c}
\hline \multicolumn{2}{c||}{$\alpha=0$} & \multicolumn{2}{c}{$\alpha=1$} \\
\hline $\max _{x \in I}\left|T_{1, \frac{1}{2}, 15}^{\alpha}[f](x)\right|$ & $\mathcal{B}_{\frac{1}{2}, 15}^{\alpha}$ & $\max _{x \in I}\left|T_{1, \frac{1}{2}, 15}^{\alpha}[f](x)\right|$ & $\mathcal{B}_{\frac{1}{2}, 15}^{\alpha}$ \\
\hline $3.52155 \times 10^{-11}$ & $7.90497 \times 10^{-10}$ & $2.2692 \times 10^{-10}$ & $1.75486 \times 10^{-8}$
\end{tabular}

EXAMPLE 4.2. Consider the function $f(z)=\cosh \left(z_{1}+z_{2}\right), z \in \mathbb{C}^{2}$, which belongs to the space $E_{0}^{2}(\varphi)$ and satisfying the exponential growth condition (2.27) on $\mathbb{R}^{2}$ with $M=1$, $\kappa=1, \sigma=0$, and $\varphi(x)=e^{x_{1}+x_{2}}$. Therefore, we can apply Corollary 3.6 with $n=2$, $N=15$, and $h=1 / 3$ for some values of $\alpha$. In this example, we use the relative errors instead of the absolute errors because $f$ has an exponential growth and the samples are exponentially increasing with respect to the real axes $\Re z_{1}$ and $\Re z_{2}$. Denote by $R_{h, N}^{\alpha}$ the relative bound associated with the real-valued bound in (3.28), i.e.,

$$
R_{h, N}^{\alpha}(x):=2 e^{\kappa\left(h+\left|x_{1}\right|+\left|x_{2}\right|\right)} \mathbb{B}_{h, N, \alpha}(0) \frac{e^{-N(\beta-\kappa h)}}{\sqrt{\pi \beta N}} / D_{x}^{\alpha} f(x),
$$

where $\alpha \in \mathbb{N}_{0}^{2}, x=\left(x_{1}, x_{2}\right) \in \mathbb{R}^{2}, \kappa$ is a non-negative number, and $\mathbb{B}_{h, N, \alpha}$ is given in (3.18). In Table 4.2, we present the approximations of the function $f$ and its mixed derivative on the domain $J=(0,3]^{2}$ with the relative errors. The graphs of the relative errors are given in Figure 4.3 for $\alpha=(0,0)$ and Figure 4.4 for $\alpha=(1,1)$. Note that $\mathfrak{T}_{n, h, N}^{\alpha}$ does not have any poles in the region $(0, \infty) \times(0, \infty)$ because $D_{x}^{\alpha} f(x)$ is either $\sinh \left(x_{1}+x_{2}\right)$ when $\alpha_{1}+\alpha_{2}$ is odd or $\cosh \left(x_{1}+x_{2}\right)$ when $\alpha_{1}+\alpha_{2}$ is even. Note that $f(z)=D_{z}^{(0,0)} f(z)$. 


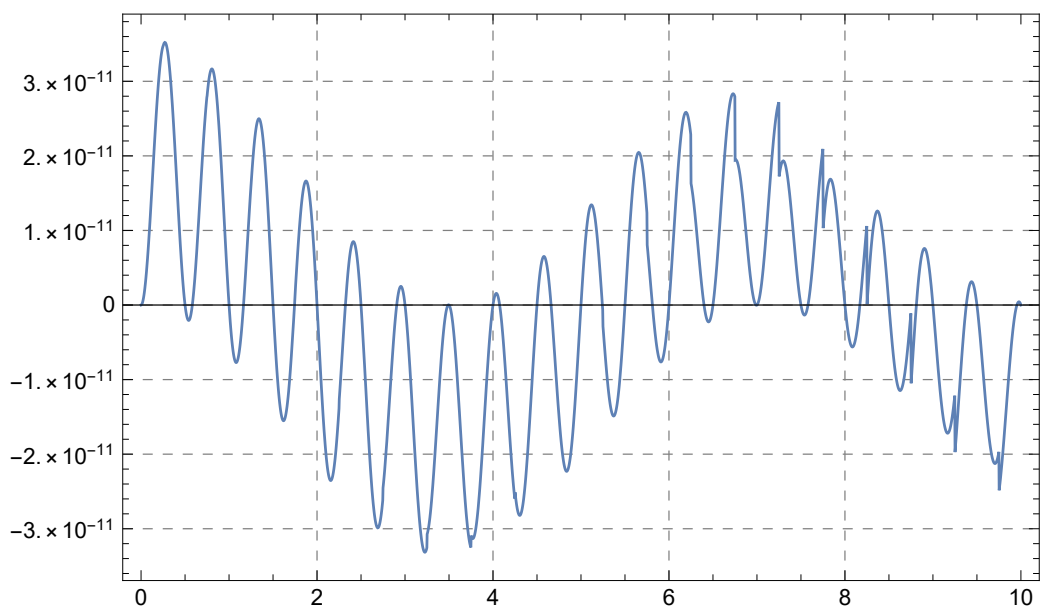

FIG. 4.1. $T_{1, \frac{1}{2}, 15}^{0}[f](x)$.

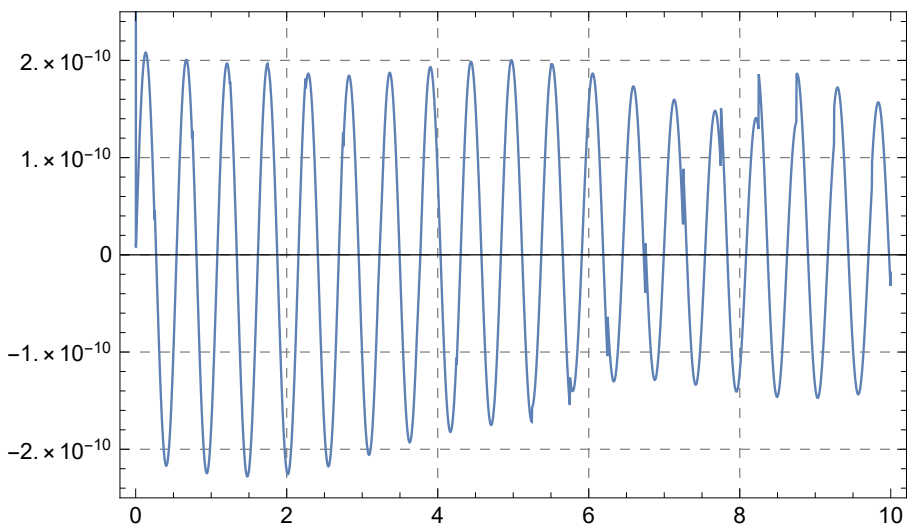

FIG. 4.2. $T_{1, \frac{1}{2}, 15}^{1}[f](x)$.

TABLE 4.2

Approximation of function $f$ and its mixed partial derivative.

Relative error for $D^{\alpha} \mathcal{G}_{n, h, N}$ with $n=2, N=15$ and $h=0.3$

\begin{tabular}{c|c||c|c}
\hline \multicolumn{2}{c||}{$\alpha=(0,0)$} & \multicolumn{2}{c}{$\alpha=(1,1)$} \\
\hline $\max _{x \in \mathcal{J}}\left|\mathfrak{T}_{2,0.3,15}^{\alpha}[f](x)\right|$ & $\max _{x \in \mathcal{J}}\left|R_{0.3,15}^{\alpha}(x)\right|$ & $\max _{x \in \mathcal{J}}\left|\mathfrak{T}_{2,0.3,15}^{\alpha}[f](x)\right|$ & $\max _{x \in \mathcal{J}}\left|R_{0.3,15}^{\alpha}(x)\right|$ \\
\hline $4.42269 \times 10^{-11}$ & $1.09931 \times 10^{-8}$ & $4.82781 \times 10^{-10}$ & $8.55546 \times 10^{-7}$
\end{tabular}

EXAMPLE 4.3. In this example, we approximate the function

$$
f(z)=\frac{\sin \left(z_{1}+z_{2}+z_{3}\right)}{\left(z_{1}^{2}+9\right)\left(z_{2}^{2}+9\right)\left(z_{3}^{2}+9\right)}, \quad z \in \mathbb{C}^{3},
$$

which belongs to the space $A_{3}^{3}(\varphi)$. Here, we can apply Corollary 3.8 with $n=3, d=3$, $N=15$, and $\varphi(x)=1 / 9^{3}$ for some values of $\alpha \in \mathbb{N}_{0}^{3}$. Let $\mathfrak{R}_{h, N}^{\alpha}$ be the relative bound 


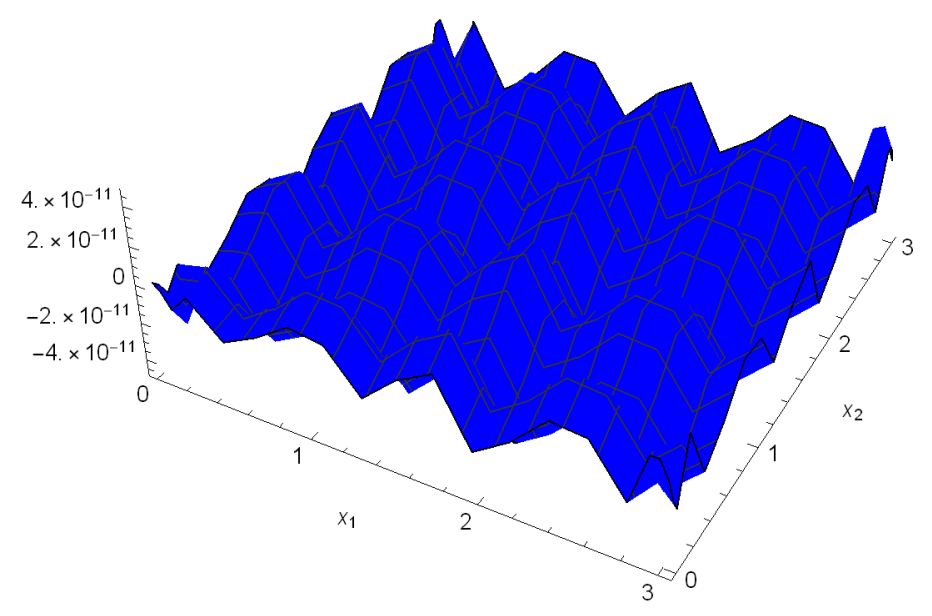

FIG. 4.3. $\mathfrak{T}_{2,0.3,15}^{(0,0)}[f](x)$.

associated with the real-valued bound in (3.33), i.e.,

$$
\mathfrak{R}_{h, N}^{\alpha}(x):=2 \sqrt{2} \varphi(\eta(x)) \mathfrak{B}_{d, N, \alpha} \frac{\mathrm{e}^{-\pi N / 2}}{\sqrt{N}} / D_{x}^{\alpha} f(x), \quad x \in \mathbb{R}^{3}, \alpha \in \mathbb{N}_{0}^{3},
$$

where $\mathfrak{B}_{d, N, \alpha}$ is defined in (3.34). In Table 4.3, we summarize the approximations of the function $f$ and its mixed derivative on the domain $\mathbf{J}=(0,2]^{3}$. Note that $f(z)=D_{z}^{(0,0,0)} f(z)$.

TABLE 4.3

Approximation of function $f$ and its mixed partial derivative.

\begin{tabular}{c|c||c|c}
\multicolumn{3}{c}{ Relative error for $D^{\alpha} \mathcal{G}_{n, \frac{d}{N}, N}$ with $n=3, N=15$ and $d=3$} \\
\hline$\alpha=(0,0,0)$ & \multicolumn{2}{c}{$\alpha=(1,1,1)$} \\
\hline $\max _{x \in \mathbf{J}}\left|\mathfrak{T}_{3, \frac{1}{5}, 15}^{\alpha}[f](x)\right|$ & $\max _{x \in \mathbf{J}}\left|\mathfrak{R}_{\frac{1}{5}, 15}^{\alpha}(x)\right|$ & $\max _{x \in \mathbf{J}}\left|\mathfrak{T}_{3, \frac{1}{5}, 15}^{\alpha}[f](x)\right|$ & $\max _{x \in \mathbf{J}}\left|\mathfrak{R}_{\frac{1}{5}, 15}^{\alpha}(x)\right|$ \\
\hline $2.9525 \times 10^{-8}$ & $9.92971 \times 10^{-7}$ & $1.44569 \times 10^{-6}$ & $1.27192 \times 10^{-4}$
\end{tabular}

5. Conclusion. In this paper, we extend the two-dimensional sinc-Gauss sampling formula, which is introduced in $[6,7]$, to the general multidimensional case. We use the multidimensional sinc-Gauss sampling formula to approximate multivariate analytic functions belonging to some wide classes of functions and their partial derivatives of any order using only finitely many samples of the function itself. This formula is valid for the class of entire functions of several complex variables satisfying a decay condition. It includes unbounded functions on $\mathbb{R}^{n}$ and a class of multivariate functions analytic in a multidimensional horizontal strip. The theoretical error analysis is established via a complex analytic approach, and the convergence rate is of exponential order. Furthermore, this paper extends the work of Lin and Zhang in [10] to the complex domains. We can use the multivariate kernel function (2.4) 


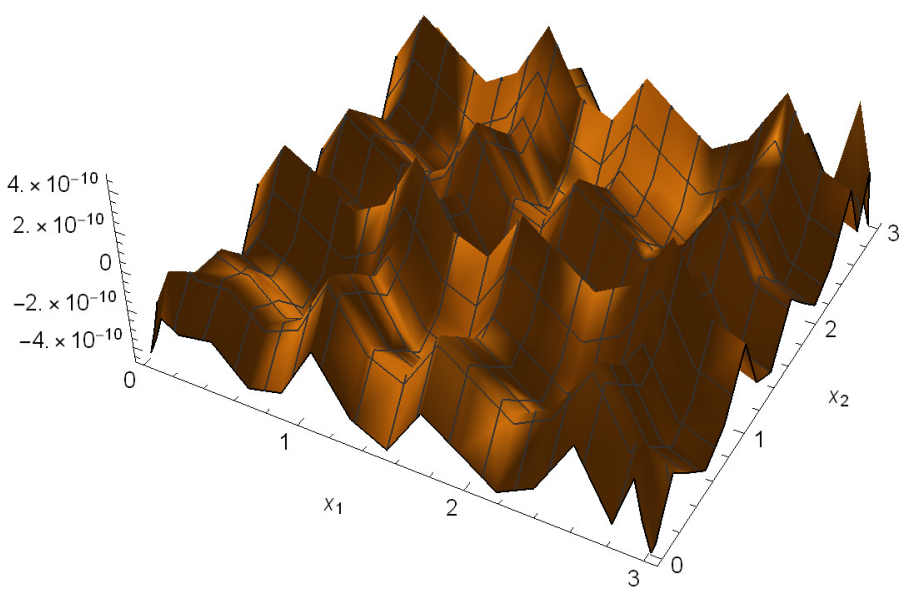

FIG. 4.4. $\mathfrak{T}_{2,0.3,15}^{(1,1)}[f](x)$.

to extend the two-dimensional Hermite-Gauss sampling formula, introduced in [3, 6], to the general multidimensional case. We recommend this for future studies.

Acknowledgement. We thank the anonymous referees for their valuable comments.

Funding. The authors would like to express their gratitudes to the ministry of education and the deanship of scientific research, Najran University, Kingdom of Saudi Arabia for their financial and technical support under code number NU/SERC/10/499.

\section{REFERENCES}

[1] L. V. Ahlfors, Complex Analysis, 3rd ed., McGraw Hill, New York, 1979.

[2] R. M. Asharabi, Generalized sinc-Gaussian sampling involving derivatives, Numer. Algorithms, 73 (2016), pp. 1055-1072.

[3] - Generalized bivariate HermiteâĂŞGauss sampling, Comput. Appl. Math., 38 (2019), Art. 29, 21 pages.

[4] - The use of the sinc-Gaussian sampling formula for approximating the derivatives of analytic functions, Numer. Algorithms, 81 (2019), pp. 293-312.

[5] R. M. ASHARABI AND F. M. AL-ABBAS, Error analysis for regularized multidimensional sampling expansion, Electron. Trans. Numer. Anal., 52 (2020), pp. 320-341.

http://etna.ricam.oeaw.ac.at/vol.52.2020/pp320-341.dir/pp320-341.pdf

[6] R. M. AShaRABi And J. PRestin, On two-dimensional classical and Hermite sampling, IMA J. Numer. Anal., 36 (2016), pp. 851-871.

[7] - Accurate sampling formula for approximating the partial derivatives of bivariate analytic functions, Numer. Algorithms, 86 (2021), pp. 1421-1441.

[8] L. C. Evans, Partial Differential Equations, 2nd ed., AMS, Providence, 2010.

[9] L. Hörmander, An Introduction to Complex Analysis in Several Variables, 3rd ed., North-Holland, Amsterdam, 1990.

[10] R. Lin AND H. Zhang, Convergence analysis of the Gaussian regularized Shannon sampling series, Numer. Funct. Anal. Optim., 38 (2017), pp. 224-247.

[11] S. M. NIKOL'SKII, Approximation of Functions of Several Variables and Imbedding Theorems, Springer, New York, 1975.

[12] L. QIAN, On the regularized Whittaker-Kotel'nikov-Shannon sampling formula, Proc. Amer. Math. Soc., 131 (2003), pp. 1169-1176. 
[13] — The Regularized Whittaker-KotelâĂŹnikov-Shannon Sampling Theorem and its Application to the Numerical Solutions of Partial Differential Equations, Ph.D. Thesis, Faculty of Science, National University of Singapore, Singapore, 2004.

[14] L. QIAN AND D.B. CREAMER, A modification of the sampling series with a Gaussian multiplier, Sampl. Theory Signal Image Process., 5 (2006), pp. 1-19.

[15] - Localized sampling in the presence of noise, Appl. Math. Lett., 19 (2006), pp. 351-355.

[16] R. M. RANGE, Holomorphic Functions and Integral Representations in Several Complex Variables, Springer, New York, 1986.

[17] V. SCheIDEmAnN, Introduction to Complex Analysis in Several Variables, Birkhäuser, Basel, 2005.

[18] G. SCHMEISSER AND F. STENGER, Sinc approximation with a Gaussian multiplier, Sampl. Theory Signal Image Process., 6 (2007), pp. 199-221.

[19] K. TANaKa, M. Sugihara, And K. Murota, Complex analytic approach to the sinc-Gauss sampling formula, Japan J. Indust. Appl. Math., 25 (2008), pp. 209-231.

[20] M. E. TAYLOR, Introduction to Analysis in Several Variables: Advanced Calculus, AMS, Providence, 2020.

[21] M. M. THARWAT, Approximation of eigenvalues of Dirac systems with eigenparameter in all boundary conditions by sinc-Gaussian method, Appl. Math. Comput., 262 (2015), pp. 113-127.

[22] J. WANG AND G. FANG, Multidimensional sampling theorem and estimate of aliasing error, Acta Math. Appl. Sin., 19 (1996), pp. 481-488.

[23] P. YE, Error bounds for multidimensional Whittaker-Shannon sampling expansion, in Proc. 2011 International Conference on Multimedia and Signal Processing, IEEE Conference Proceedings, Los Alamitos, 2011, pp. 33-36. 